\title{
Characterizing Collaborative 4D Use Contexts to Improve Interaction Mechanisms Design
}

\author{
Conrad Boton $^{1}$, Gilles Halin ${ }^{2}$ and Sylvain Kubicki ${ }^{1}$ \\ ${ }^{1}$ Public Research Centre Henri Tudor. 29, avenue JF Kennedy, L-1855, Luxembourg- \\ Kirchberg, PH (352) 425991 - 1; FAX (352) 425991 - 777; email: \\ conrad.boton@tudor.lu, sylvain.kubicki@tudor.lu \\ ${ }^{2}$ CRAI - Research Centre in Architecture and Engineering. 2, rue Bastien Lepage. \\ 54001 Nancy, France; PH (33) 3833081 46; FAX (33) 3833081 27; email: \\ gilles.halin@crai.archi.fr
}

\begin{abstract}
This article proposes a metamodel to describe the use context of collaborative 4D modeling in Architecture, Engineering and Construction. While interaction with three-dimensional models has been extensively discussed in the literature, the interaction principles with the fourth dimension are much less treated. It is indeed a challenging issue according to the characteristics of the construction industry. The proposed metamodel is a preliminary step toward the proposal of adapted and/or innovative visualization and interaction mechanisms in collaborative 4D tools. It allows a good understanding of the collaborative 4D simulation activity and the identification of the main variables to consider from a high level point of view.
\end{abstract}

\section{INTRODUCTION}

This paper presents first results of a research work related to the identification of the best visualization and interaction mechanisms in collaborative 4D simulation tools. The work is part of a more comprehensive framework that aims to adapt the visualization to the business needs of the users. This is a challenging issue because of the peculiarities of construction industry and special planning practices traditionally used. It should be recalled that the construction industry is characterized by a lot of fragmentation (Howard et al. 1989; Sun \& Aouad 2000). Each construction project implements a particular and always different collaboration context, and the environment in which construction projects take place (e.g., the physical context, legal jurisdictions, market conditions, and management mechanisms) is always different and in constant change. Each project sets up a different collaboration context with actors who do not always play the same roles.

Moreover, in terms of visualization, practitioners are used to some business views. Business views are defined as views that professionals handle in their everyday work (Kubicki et al. 2007). Whether via paper or digital media, they are accustomed to make use of representation techniques that enable them to better respond to their need for visualization. Several views may represent the same concept 
and professionals used to choose one or the other depending on their specific information needs. For example, if a Gantt chart and a PERT network can both represent planning, PERT network is more relevant to show the critical path. Adapting visualization and interaction to the practitioners' business needs in 4D tools is therefore to understand their needs and to choose the most appropriate representation and interaction principles to meet these needs, based as much as possible on the business views.

In this sense, the first part of the research work had proposed a method and metamodels for describing the context of the activity, for choosing the most appropriate business views and for coordinating them (Boton et al. 2012; Boton et al. 2010; Halin et al. 2011). But once the views are proposed, it is necessary to associate them with appropriate interaction mechanisms to enable users to interact with them in the best way. But if interaction with three-dimensional models has been extensively discussed in the literature (Grossman et al. 2001; Hand 1997; Herndon et al. 1994), the interaction principles with the fourth dimension are much less treated (Yan et al. 2012). Moreover, a variety of devices are nowadays available with different interaction capabilities. This paper proposes a description of the collaborative 4D modeling, in order to identify the variables to consider in the choice of interaction mechanisms when designing specific 4D applications.

\section{RELATED WORKS}

The issue of interaction in 4D models is not really new. Already in 1999, Liston identified three aspects to consider in the design of appropriate 4D representations (Liston 1999). The first of these aspects was the definition of appropriate interaction mechanisms, firstly among the 4D representation elements, and also between the 4D content and the users. She then classified the content into three categories: descriptive content (related to the description of the process, such as work areas, construction areas), explanatory content (related to the logic of scheduling, for example, why an activity precedes another) and predictive content (related to the behaviour of a specific sequence, such as cost, time or productivity). This categorization is probably the basis of the types of visualization tasks identified later by Tory and Staub-French: descriptive tasks, explanatory tasks, evaluative tasks and predictive tasks (Tory \& Staub-French 2008).

In 2002, Waly and Thabet proposed a virtual construction environment (VCE) for pre-construction planning. To this end, they introduced an Interactive Virtual Interface (IVI), defined as a dynamic virtual system allowing the project team to take over the fourth dimension and simulate the construction process in a fairly realistic way. In this virtual interface, users can graphically "drag and drop" elements from the 3D model and rebuild the building by putting side by side the components in the order received for the actual construction. The IVI offers intuitive interaction mechanisms and interesting such as 'click to take' or 'release to place' or 'navigate to the walk through' (Waly \& Thabet 2002).

CIFE (Center for Integrated Facility Engineering) from the University of Stanford developed the iRoom, an integrated system allowing 4D-based collaboration among different construction industry practitioners (Fischer et al. 2002). It consists of 
a server and multiple projectors connected to computers and large screens. The screens can be viewed simultaneously and the system includes multiple applications (MS Project, Excel, 4D modelling, etc.) and viewers. Users can then view a 4D scenario on different screens simultaneously. To increase the perception of users, two different visualization techniques (highlight and overlay) are prototyped, evaluated and patented. Highlight here is defined as the process of light through a visual annotation of sets of related information in a view or across multiple views. Overlay consists in placing a set of information on another set of data, which gives a "merged" view (Liston \& Fischer 2000).

In 2005, Rischmoller and Valle observed that the interactions used in the traditional 4D simulation approach are not always relevant to display certain aspects of the construction. They made the hypothesis that a 3D model is not a mandatory requirement in the construction of a 4D model, and proposed a new conceptualization of 4D. This conceptualization uses digital 2D tables that can display dynamically as rows and columns arranged in a special way, the start and end dates for each subcontractor tasks (Rischmoller \& Valle 2005).

In 2009, Zhou et al. proposed an interactive method for 4D model definition. The method uses a 3D model as starting point and provides an opportunity for multidisciplinary actors to focus on this common illustration of design (represented by the $3 \mathrm{D}$ model) in order to analyze the design, discuss the planning strategies and examine possible solutions. The question of visualization interaction is not very explicitly considered but an interactive collaboration workflow is proposed. It is based on collaborative sessions and identifies the features required for a collaborative interactive 4D tool (Zhou et al. 2009).

With the advent of new devices (smartphones, tablets, etc.) that are becoming increasingly used in construction projects, interactions adaptation has to take into account the opportunities offered by these devices. Yan et al. (2012) recently introduced the multitouch interfaces as interesting environments for intuitive exploration of the fourth dimension geometric concepts. They then proposed a multitouch interface mechanism to interact with the 4D geometry, more intuitive and easier to learn than traditional mouse-based interactions (Yan et al. 2012).

More recently, Boton et al. (2013) proposed a conceptualization of collaborative 4D simulation. This conceptualization is partly derived from simulation theories and showed the different roles of the involved actors (Boton et al. 2013): the doer (the one who performs the simulation), the done for (the one for which the simulation is performed), the done with (members of the simulation team), the done to (those who provide the necessary information for the simulation), and the done without (those who do not participate in the simulation, but are nevertheless directly interested in the results).

These works are very interesting and are important milestones towards the adapted interaction techniques in collaborative 4D simulation tools. But to be able to link the interaction principles with business needs, it is necessary to have a broader discussion involving not only the theories of 4D simulation, visualization and humancomputer interaction, but also a good understanding of business situations and theories on Computer-Supported collaborative Work (CSCW). 


\section{USE CONTEXTS DESCRIPTION}

To describe collaborative 4D use contexts, it is firstly necessary to define a structured language. The aim is to ensure that the contexts are described in a single way, so they are comparable and reusable. Model Driven Engineering (MDE) approach recommends the use of metamodels to define domain languages (Favre et al. 2006). This approach enables the design of models which have to be conformed to their metamodel. Applied to our issue, before being able to create use context models, we need first to define a use context metamodel. According to our particular issue, we consider that a collaborative 4D simulation involves multiple users, manipulates one or more artifacts and uses a particular simulation approach.

Each user works in a physical context and has a type and an operational role. The physical context is related to the localization (office, building site, mobility) and the type of device used (desktop, laptop, tablet, smartphone, etc.). The type of users is related to the role they play in the construction project context (architect, engineer, owner, contractor, surveyor, manager, etc.) while the operational role is the role they have in the simulation process (doer, done to, done for, done with, done without). Based on these different roles, the actor performs some individual practices that will greatly influence his interaction needs.

The artefact is described by the type of construction (residential building, commercial building, institutional building, light industrial building, road construction, or high mass construction) and the type of content (descriptive, explanative, evaluative, or predictive).

The simulation approach involves not only the approach to creation of the 4D model, but also the style of work of the group and its space-time distribution. Zhou et al. (2009) identified three main approaches used to produce a 4D simulation: manual linking, automation and manual assembly. In manual linking approach, the relationship between the $3 \mathrm{D}$ model and the activities scheduling is done manually using third party software. In automation approach, the link between the Product Breakdown Structure and the Work Breakdown Structure is automated in order to generate an automatic link between the 3D model and the planning. The manual assembly approach suggests using the 4D simulation for initial planning and not only for planning review. The interest is to interactively build a schedule from a 3D model of the building.

The space-time classification comes from CSCW theories (Johansen 1988). It classify the dispersion of the participants using a matrix to compare the time and place of cooperation. On the one hand, there are synchronous meetings (same time) where participants are simultaneously present, and asynchronous meetings where participants work on different moments. On the other hand, we have meetings faceto-face (same place) and electronic meetings (different places). The combination of the two parameters (time and space) gives four situations: colocated synchronous, colocated asynchronous, distributed synchronous, distributed asynchronous.

About the style of organization in construction projects, it was discussed in the previous works. Kubicki identified three different styles of organization: hierarchical organization, adhocratic organization and transversal organization (Kubicki 2006). These types of organizations will influence the level of flexibility in 
the proposed interaction. For example, in an adhocratic organization where mutual adjustment is paramount, it is possible to imagine interaction mechanisms that are reconfigurable by actors themselves according to their needs. Instead, in a hierarchical organization, the interactions can be more easily imposed to users by their immediate supervisor.

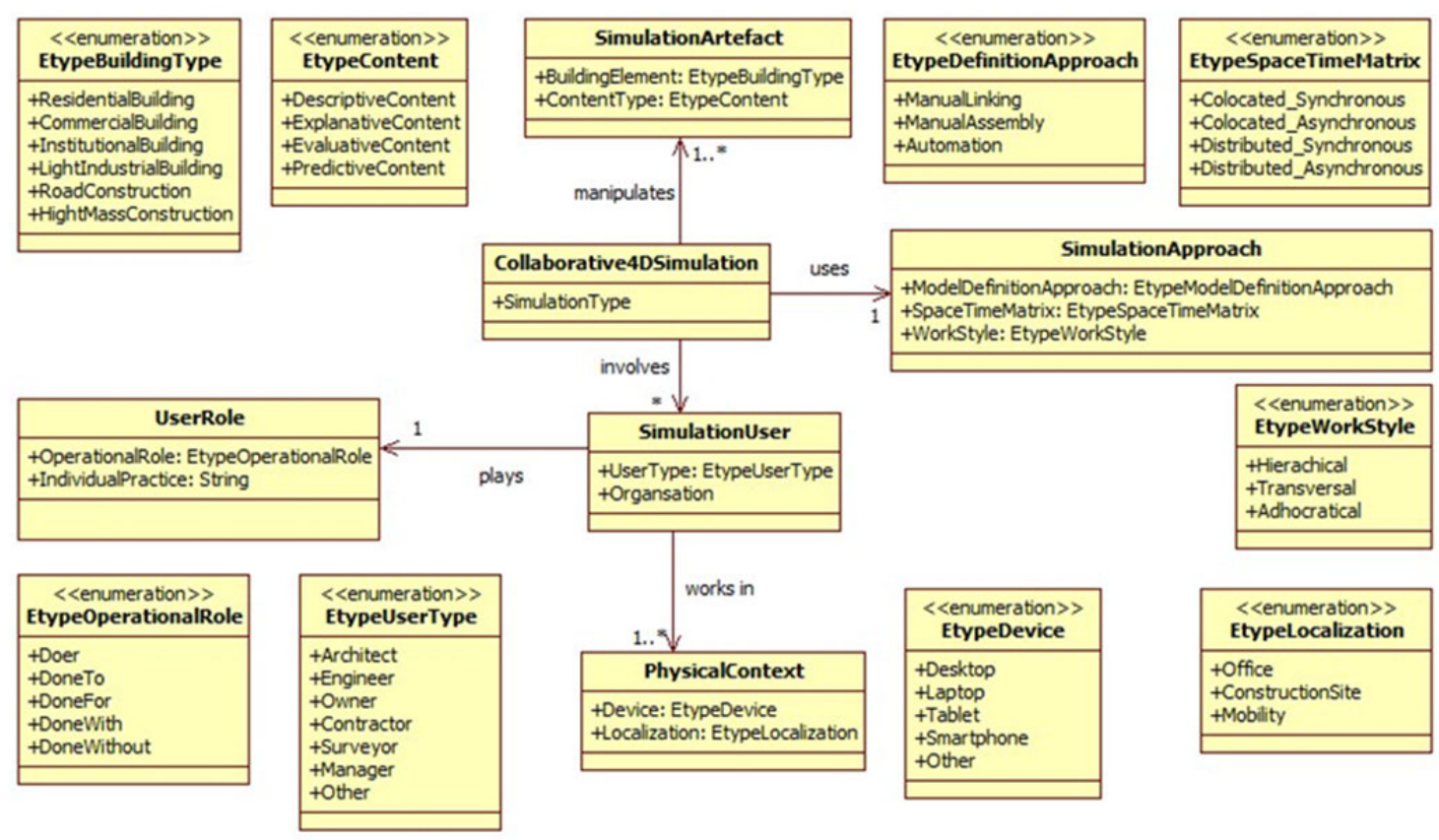

Figure 1. Metamodel of collaborative 4D use context.

\section{PROOF OF CONCEPT}

To demonstrate the use of the proposed metamodel, we take three examples of collaborative 4D simulation based on elements from literature. Tables 1, 2 and 3 show the description of the use context of this collaboration, on the basis of the proposed metamodel.

The first example considers a collaborative 4D simulation based on the iRoom system. This example stages an architect and owner collaborating via the iRoom on the planning of a residential building. We assume that the architect generated automatically a 4D simulation using specific software, in order to illustrate the construction sequences. He plays the model to allow the owner to understand the construction process and validate it.

In the second example, two different subcontractors are working to virtually reconstruct a residential building with in the VCE environment. Their aim is to identify potential conflicts and clashes, and to optimize the construction areas. They then reconstruct manually the building elements using the interaction mechanisms enabled by the VCE system. The first subcontractor comes from an Organization A while the second subcontractor belongs to an organization $\mathrm{B}$. The starting point of their work is a first draft of $4 \mathrm{D}$ sequences. The work takes place in an office where the VCE system is implemented. 
The third example is based on the definition method described by Zhou et al. (2009). In this example, a supervisor is responsible of a road construction site. From his office, he is working to provide the contractor with a 4D model to support his site work. He creates the model manually and sends it to the contractor. Later, the contractor will use the model at the construction site, to better understand his work and will provide some feedbacks to improve the model for the future similar works. The supervisor belongs to an organization $\mathrm{C}$ and the contractor belongs to an organization D. In the framework of the construction project, the supervisor is the immediate supervisor of the contractor.

\section{Table 1. Description of an iRoom-based simulation use context}

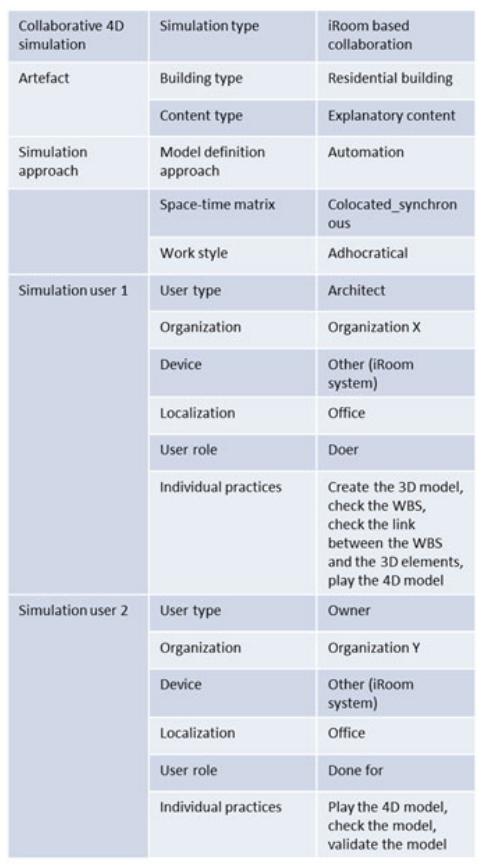

\section{Table 2. Description of a VCE-based simulation use context}

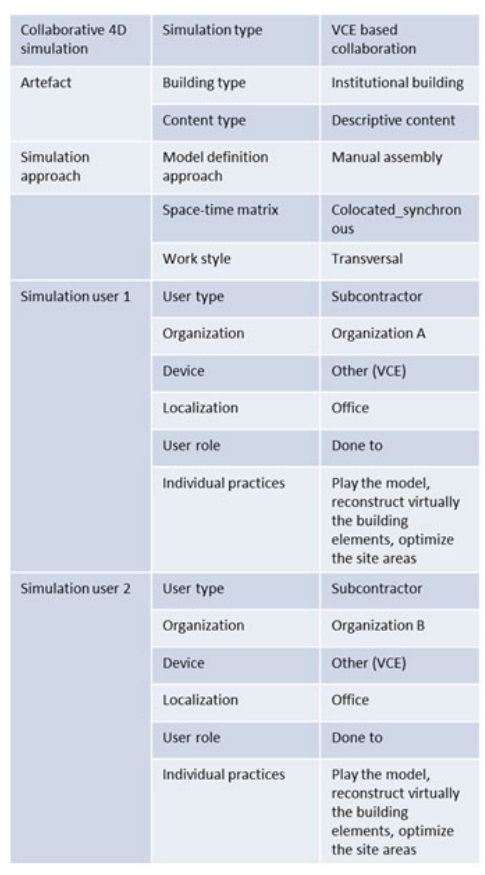

Table 3. Description of a distributed simulation use context

\section{CONCLUSION}

Through the three examples presented above, it appears that the design of adapted interaction principles in collaborative 4D simulation tools should consider at least a certain number of variables. Among these variables, we identified:

- the artefact: this encompasses not only the type of the construction project, but also the type of content that is manipulated;

- the simulation approach: this is related to the 4D model definition approach, the space-time distribution of the group, and the its work style;

- the users: the user description should take into account their type, the device they work on, their localization, the role they play in the simulation process, and their individual practices. 
This work is a very important starting point because it proposes a good understanding of the collaborative 4D simulation activity. It also provides variables to consider when designing adapted visualization and interaction mechanisms in collaborative 4D simulation systems. Moreover, any adaptation work can then be positioned clearly and be compared to another one.

Future works will make the link between these variables, business views and interaction mechanisms. Such link will enable the proposal of guidelines to support the interaction design work.

\section{REFERENCES}

Boton, C., Halin, G., \& Kubicki, sylvain. (2012). A metamodel to describe nD CAD visualization as Coordinated Multiple Views. In Yuhua Luo (Ed.), Lecture Notes in Computer Science, Volume 7467/2012. Springer Berlin Heidelberg, 219-226.

Boton, C., Kubicki, S., \& Halin, G. (2010). Adaptation of user views to business requirements: towards adaptive views models. In Conference Internationale Francophone sur I'Interaction Homme-Machine, ACM, 113-116.

Boton, C., Kubicki, S., \& Halin, G. (2013). Collaborative 4D/nD construction simulation: What is it? In Y. Luo (Ed.), Lecture Notes in Computer Science. Mallorca: Springer Berlin Heidelberg, 161-168.

Favre, J.-M., Estublier, J., \& Blay-Fornarino, M. (2006). L’ingénierie dirigée par les modèles au-delà du MDA, Lavoisier, Paris, France. 227 p.

Fischer, M., Stone, M., Liston, K., Kunz, J., \& Singhal, V. (2002). Multi-stakeholder collaboration: The CIFE iRoom. International Council for Research and Innovation in Buildig and Construction. CIB w78 conference, Aarhus School of Architecture, 12-14.

Grossman, T., Balakrishnan, R., Kurtenbach, G., Fitzmaurice, G., Khan, A., \& Buxton, B. (2001). Interaction techniques for 3D modeling on large displays. Proceedings of the 2001 symposium on Interactive 3D graphics, New York, USA: ACM, 17-23.

Halin, G., Kubicki, S., Boton, C., \& Zignale, D. (2011). From Collaborative Business Practices to User's Adapted Visualization Services: Towards a Usage-Centered Method Dedicated to the AEC Sector. In Y. Luo (Ed.), Lecture Notes in Computer Science, Volume 6874, Berlin, Heidelberg: Springer Berlin Heidelberg, 145-153.

Hand, C. (1997). A Survey of 3D Interaction Techniques. Computer Graphics Forum, 16(5), 269-281.

Herndon, K. P., Van Dam, A., \& Gleicher, M. (1994). The challenges of 3D interaction: a CHI '94 workshop. SIGCHI Bull., 26(4), 36-43.

Howard, H., Levitt, R., Paulson, B. C., Pohl, J. G., \& Tatum, C. B. (1989). Computer integration: reducing fragmentation in AEC industry. Journal of Computing in Civil Engineering, 3(1), 18-32.

Johansen, R. (1988). Groupware: Computer support for business teams? New York, NY, USA: The Free Press, 205 p.

Kubicki, S. (2006). Assister la coordination flexible de l'activité de construction de bâtiments, Une approche par les modèles pour la proposition d'outils de 
visualisation du contexte de coopération. Thèse de doctorat. Université Henri Poincaré, Nancy I.

Kubicki, S., Halin, G., \& Guerriero, A. (2007). Multi-visualization of the Cooperative Context in Building Construction Activity A Model-Based Approach to design AEC-specific Visualization Interfaces. 2007 11th International Conference Information Visualization (IV'07), 590-595.

Liston, K., \& Fischer, M. (2000). Designing and evaluating visualization techniques for construction planning. Computing in Civil and Building.

Liston, K. M. (1999). Designing 4D contexts for construction planners. CHI '99: CHI '99 extended abstracts on Human factors in computing systems , 65-66. New York, NY, USA: ACM.

Rischmoller, L., \& Valle, E. (2005). Using 4D in a new “ 2D + time ” Conceptualization. In Raimar, J. Scherer, P. Katranuschkov, \& S.-E. Schapke (Eds.), Proceedings of CIB 78 22nd Conference on Information Technology in Construction, Dresden Germany: Institute for Construction Informatics, 247251.

Sun, M., \& Aouad, G. (2000). Integration technologies to support organisational changes in the construction industry. 7th ISPE International conference on Concurrent Engineering, Lyon, France, 596-604.

Tory, M., \& Staub-French, S. (2008). Qualitative analysis of visualization: a building design field study. In ACM (Ed.), Proceedings of BELIV, Florence, Italy.

Waly, A. F., \& Thabet, W. Y. (2002). A Virtual Construction Environment for preconstruction planning. Automation in Construction, 12, 139-154.

Yan, X., Fu, C.-W., \& Hanson, A. J. (2012). Multitouching the fourth dimension. Computer, 45(9), 80-88.

Zhou, W., Heesom, D., Georgakis, P., Nwagboso, C., \& Feng, A. (2009). An interactive approach to collaborative $4 \mathrm{~d}$ construction planning. Journal of Information Technology, 14(March), 30-47. 